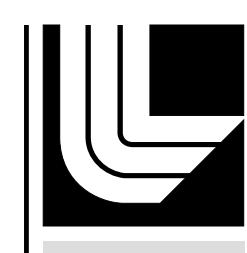

LAWRENCE LIVERMORE N A T IO N A L LABORATORY

\title{
Modeling non-equilibrium phase transitions in isentropically compressed $\mathrm{Bi}$
}

Jave Kane, Raymond Smith

September 27, 2005

14th APS Topical Conference on Shock Compression of Condensed Matter Baltimore, MD, United States August 1, 2005 through August 5, 2005 
This document was prepared as an account of work sponsored by an agency of the United States Government. Neither the United States Government nor the University of California nor any of their employees, makes any warranty, express or implied, or assumes any legal liability or responsibility for the accuracy, completeness, or usefulness of any information, apparatus, product, or process disclosed, or represents that its use would not infringe privately owned rights. Reference herein to any specific commercial product, process, or service by trade name, trademark, manufacturer, or otherwise, does not necessarily constitute or imply its endorsement, recommendation, or favoring by the United States Government or the University of California. The views and opinions of authors expressed herein do not necessarily state or reflect those of the United States Government or the University of California, and shall not be used for advertising or product endorsement purposes. 


\title{
Modeling non-equilibrium phase transitions in isentropically compressed $\mathrm{Bi}$
}

\author{
J. O. Kane ${ }^{1}$, R. F. Smith ${ }^{2}$ \\ ${ }^{1}$ Lawrence Livermore National Laboratory, L-473, 7000 East Avenue, Livermore CA 94551 \\ ${ }^{2}$ Lawrence Livermore National Laboratory, L-286, 7000 East Avenue, Livermore CA 94551
}

\begin{abstract}
We report here on modeling of non-equilibrium phase transitions in $\mathrm{Bi}$ samples isentropically compressed to $120 \mathrm{GPa}$ by a ramped drive, which is produced using the Janus laser. In the experiments, the Bi samples are attached to windows of LiF or sapphire, and the velocity history of the sample-window interface is recorded with line VISAR. The 1D response of the targets is modeled using a multiphase Bi EOS, the Andrews-Hayes method for non-equilibrium transitions, and a Boettger-Wallace kinetics model. The pressure drive is deduced by back integration of VISAR data from shots performed with $\mathrm{Al}$ samples.
\end{abstract}

Keywords: Phase transitions, rate dependence, Bi, Andrews-Hayes, laser ICE, isentropic compression.

PACS: $64.70 . \mathrm{Kb}, 64.70 . \mathrm{Dv}$.

\section{INTRO}

Isentropic compression experiments (ICE) have been performed with a variety of drivers, including pulsed power machines, gas guns, and lasers [1, 2, 3, 4]. At the strain rates produced in a compressed sample in any of these experiments, first-order phase transitions are easily overdriven. Furthermore, the strain rates produced in an isentropically compressed sample can vary by orders of magnitude across these different experimental facilities.

As a result, the pressure and temperature trajectories of a compressed sample can deviate significantly from the equilibrium isentrope, and the particle velocity of the sample material can exhibit rate-dependence effects. Therefore, modeling of wave propagation in the compressed sample must account for non-equilibrium phase transitions and involve rate-dependent kinetics.

Recently, Bi ICE experiments were performed at the Sandia $\mathrm{Z}$ machine [1]. In the present discussion we present modeling of Bi ICE experiments performed at the Janus laser at
Lawrence Livermore National Laboratory. One goal of the experiments modeled here is to allow cross-platform study of rate-dependence in nonequilibrium phase transitions of $\mathrm{Bi}$ and other materials.

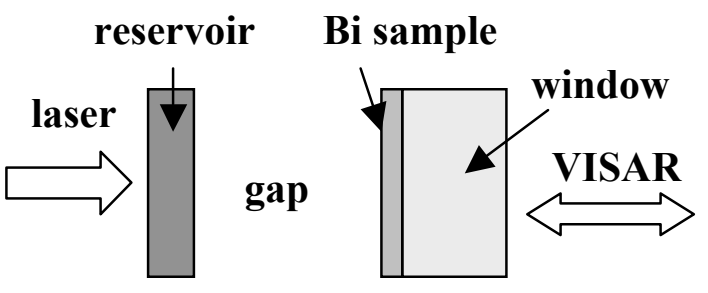

Figure 1. Experiment. The reservoir is $75 \mu \mathrm{m}$ polyimide, the gap width is $425 \mu \mathrm{m}$, and the Bi samples are from $10-30 \mu \mathrm{m}$ thick. The on-target intensity was $3.8 \times 10^{12}$ $\mathrm{W} / \mathrm{cm}^{2}$ in a $2 \mathrm{~ns}$ square pulse of $527 \mathrm{~nm}$ light.

\section{EXPERIMENTAL PROCEDURE}

The Janus experiment is depicted schematically in Fig. 1. The sample is loaded with a ramped laser ICE drive. The drive is produced 
when shock loaded reservoir material is released across a gap and then stagnates on the gap-facing side of the sample. In the present experiments, the peak pressure of the drive is $12 \mathrm{Gpa}$ (120 kbar). The velocity history of the sample-window interface is recorded using line $\operatorname{VISAR}[5,6]$. A cartridge heating system is employed to select the initial temperature (between room temperature and melting) of the sample. The experiment is described in detail in [7].

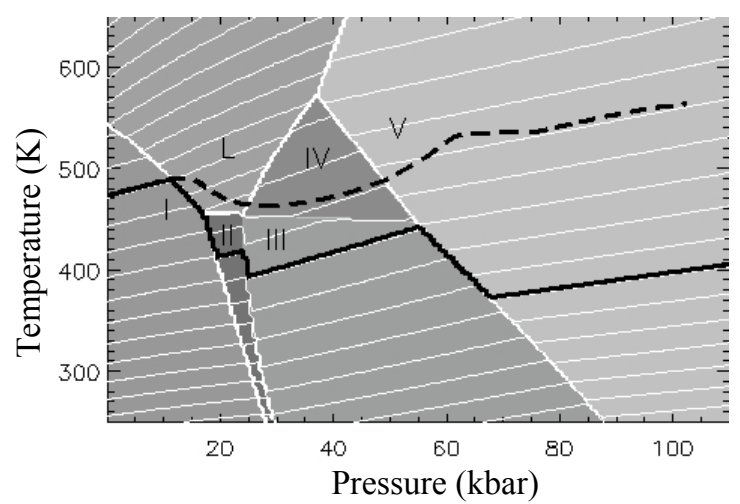

Figure 2. Bi EOS used for present modeling. Shaded regions: stability fields of indicated phases. White lines: equilibrium isentropes. Solid black line: equilbrium isentrope through atmospheric pressure and $200{ }^{\circ} \mathrm{C}$. Dashed black line: non-equilibrium model trajectory of $\mathrm{Bi}$ zone next to window, with Bi initially at atmospheric pressure and $200{ }^{\circ} \mathrm{C}$.

\section{MODELING}

To model $1 \mathrm{D}$ wave propagation in the $\mathrm{Bi}$ samples, we use the Andrews-Hayes method [8, 9, $10]$ to advance material zones in $(P, T, \boldsymbol{x})$ space, where $P$ is pressure, $T$ is temperature, and the components of the vector $\boldsymbol{x}$ are the mass fractions of the individual phases. This method assumes that all phases within a zone are individually in thermodynamic equilibrium, and all phases are at a common $P, T$. Within a zone, extensive quantities such as energy and entropy are the mass fractionweighted sums of the quantities corresponding to the individual phases. We do not model strength, or effects of grain size and orientation. Steep waves are stabilized by Von Neumann quadratic and linear artificial viscosity.

In the modeling, we apply a pressure drive to the gap-facing side of the target. The drive is deduced by back integration [11] of VISAR data from shots performed with Al samples in place of the Bi.

The Bi EOS (Fig. 2) contains five solid phases and a liquid phase. Phases I, II and the liquid are the Johnson Bi EOS [12]. Phases III, IV and V have the same form as phase II, with Johnson parameters chosen to reproduce roughly phase boundaries, Clapeyron slopes, volume changes and cold curves prescribed in $[13,14,15]$.

In the present discussion, we use a BoettgerWallace [16] kinetics model, which balances the Gibbs driving force with a reverse stress due to the volume change, creating 'metastable surfaces' that essentially replace the equilibrium $(P, T)$ phase lines.

The experimental trajectories potentially involve numerous stable and metastable phase transformations. Since there is little guidance in seeking a detailed multi-parameter BoettgerWallace kinetics model, for simplicity we set all rate constants $\tau$ to a single value, all activation energies $A_{\mathrm{F}}$ to zero, and all energy scale parameters $B_{\mathrm{F}}$ to a single value.

We model the LiF using a Mie-Gruneisen EOS.

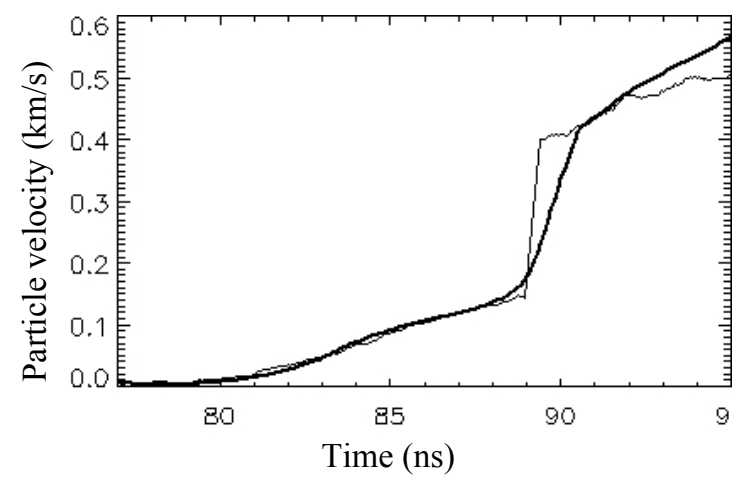

Figure 3. Modeling (heavy line) vs. VISAR data for Bi next to window, with $\mathrm{Bi}$ initially at atmospheric pressure and $200{ }^{\circ} \mathrm{C}$.

\section{RESULTS}

In Fig. 3 we compare a typical piece of VISAR-derived particle velocity data to our modeling. The $\mathrm{Bi}$ sample was initially at atmospheric pressure and a temperature of $200^{\circ} \mathrm{C}$. 
The window was LiF. The particle velocity history shows a familiar two-wave structure, with a 'plateau' and subsequent rise. In Fig. 2 we show the predicted $(P, T)$ trajectory of the $\mathrm{Bi}$ immediately adjacent to the window, as well as the equilibrium isentrope derived from the EOS.

In Fig. 2, the model trajectory is initially isentropic. However, once the phase I stability field is exited, a non-equilibrium mixture of phases is present, and in $(P, T)$ space the material follows a non-equilibrium isentrope instead of the equilibrium isentrope. The particular nonequilibrium isentrope followed depends upon the compression history of the material, the equation of state, and the kinetics model. The steep rise in particle velocity produces entropy, after which the material cannot return to the equilibrium isentrope.

As Fig. 3 shows, the model reproduces features of the observed VISAR particle velocity record, including the sloped plateau and the subsequent rise. Equilibrium modeling — with infinitely fast transition to the phase having lowest Gibbs free energy (not shown) predicts a flat plateau; see [1] for another perspective on the slope of the plateau. Generally for these experiments, the rise in the data has a more abrupt onset and a steeper slope (shock-like) than the modeling predicts. Finding the source of this disagreement may be useful in evaluating candidate EOS's, the applicability of the Andrews-Hayes method (see discussions in $[9,10]$ ), and possible effects related to mechanisms of elastic or plastic deformation.

It should also be noted that while a two-wave structure occurs naturally when there is a single phase transition, for these experiments, the modeling predicts that the material trajectory will cross more than one equilibrium phase boundary, as well as various metastable boundaries. Thus, in general a multi-wave structure is potentially possible, and its genesis may involve both stable and metastable transitions. For examples and discussion relevant to these points, see [9].

Therefore, in the present modeling, the apparent two-wave structure is a complex product of the EOS, the kinetics model, and the compression history. Furthermore, reflected waves at the sample-window interface somewhat modify the trajectory from the in-situ (no window) trajectory.

\section{CONCLUSIONS}

We have reported on modeling of laser ICE experiments in which $\mathrm{Bi}$ was isentropically compressed to $12 \mathrm{Gpa}$ starting from atmospheric pressure and initial temperatures between room temperature and melting. The very simple nonequilibrium modeling and multi-phase $\mathrm{Bi}$ EOS discussed here appear to qualitatively reproduce features of the VISAR data.

\section{ACKNOWLEDGEMENTS}

This work was performed under the auspices of the U.S. Department of Energy by the University of California, Lawrence Livermore National Laboratory under Contract No. W-7405-Eng-48.

\section{REFERENCES}

1. M. Bastea, S. Bastea, J.A. Emig, P.T. Springer, D.B. Reisman, "Kinetics of propagating phase transformation in compressed bismuth", Phys. Rev. B, 71, 180101(R), (2005).

2. L.C. Chhabildas and L.M. Barker, Sandia Report SAND86-1888 (1986)

3. J.R. Asay, Int. J. Impact Engng. 20, 27 (1997)

4. J. Edwards et al., Phys. Rev. Lett., 92 (7), 075002 (2004).

5. Barker, L. M., and Hollenback, R. E., "Laser Interferometer for Measuring High Velocities of any Reflective Surface", J. of Applied Physics 43, 4669, 1972.

6. Bloomquist, D. D. and Sheffield, S. A., "Optically recording interferometer for velocity-measurements with subnanosecond resolution," Journal of Applied Physics, vol. 54, no. 4, pp. 1717-1722, 1983

7. Smith, R. F. et al., in preparation for submittal to Physical Review Letters.

8. D.J. Andrews, J. Comp. Phys. 7, 310-326 (1971).

9. D.B. Hayes, J Appl. Phys. 46, 3438-3443 (1975).

10. Duvall, G. E. Duvall and R. A. Graham, Rev. Mod. Phys., 49, 523 (1977)

11. D.B. Hayes, Backward Integration of Equations of Motion to Correct for Free Surface Perturbations, Sandia Report SAND2001-1440 (2001).

12. J. N. Johnson et al., J. Phys. Chem. Solids 35, 501 (1974).

13. Klement et al., PR 131 (2), 632 (1963).

14. P. W. Bridgman, PR 48, 893 (1935). 
15. M. I. McMahon, O. Degtyareva, and R. J. Nelmes,PRL 854896 (2000).

16. Boettger and Wallace, Phys. Rev. B, 55 (5), 2840 (1997). 